TRANSACTIONS OF THE

AMERICAN MATHEMATICAL SOCIETY

Volume 356, Number 10, Pages 3869-3880

$\mathrm{S} 0002-9947(03) 03424-\mathrm{X}$

Article electronically published on November 25, 2003

\title{
QUANTUM DEFORMATIONS OF FUNDAMENTAL GROUPS OF ORIENTED 3-MANIFOLDS
}

\author{
UWE KAISER
}

\begin{abstract}
We compute two-term skein modules of framed oriented links in oriented 3-manifolds. They contain the self-writhe and total linking number invariants of framed oriented links in a universal way. The relations in a natural presentation of the skein module are interpreted as monodromies in the space of immersions of circles into the 3-manifold.
\end{abstract}

\section{INTRODUCTION}

Let $M$ be a compact oriented 3-manifold (possibly with boundary). Throughout "link" will mean framed and oriented link in $M$.

In [13] (see also [9]) J. Przytycki defines the quantum deformation of $\pi_{1}(M)$ in the following way: Let $\mathfrak{L}(M)$ be the set of isotopy classes of links in $M$. Let $\mathcal{S}(M)$ (in [13] denoted by $\mathcal{S}^{f r}(M)$ ) be the quotient of the free $\mathbb{Z}\left[q^{ \pm 1}\right]=: R$-module with basis $\mathfrak{L}(M)$ by the relations

$$
K_{+}=q^{2} K_{-} \quad \text { and } \quad K^{(1)}=q K .
$$

Here $K_{ \pm}$are two links, which differ only inside a 3-ball in $M$ by a crossing change. The link $K^{(1)}$ is defined from $K$ by introducing a positive twist into the framing of one of its components. The pictures below show projections onto a plane contained in some oriented 3 -ball in $M$. We assume that framings are induced from the projection plane.

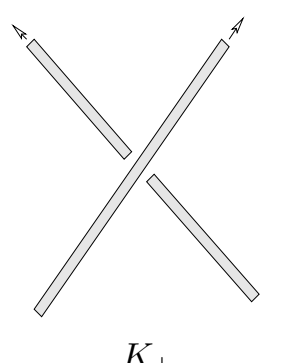

$K_{+}$

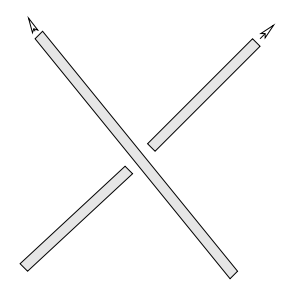

$K_{-}$

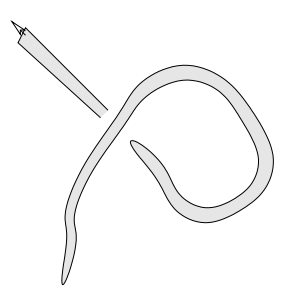

$K^{(1)}$

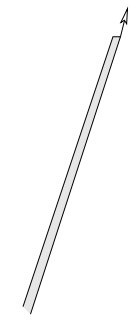

$K$

Let $\hat{\pi}(M)$ be the set of conjugacy classes of the fundamental group $\pi_{1}(M)$. For each ring $\mathcal{R}$ let $S \mathcal{R} \hat{\pi}(M)$ be the symmetric algebra of the free $\mathcal{R}$-module with basis $\hat{\pi}(M)$.

Received by the editors March 12, 2002 and, in revised form, May 1, 2003.

2000 Mathematics Subject Classification. Primary 57M25, 57M35, 57R42.

(C)2003 American Mathematical Society 
Theorem 1 (Przytycki). (a) If $M$ has no non-separating 2-spheres and tori, then $\mathcal{S}(M)$ is isomorphic to $S R \hat{\pi}(M)$.

(b) If $M$ contains a non-separating 2-sphere or torus, then $\mathcal{S}(M)$ has a torsion element:

(i) If $K$ is a link in $M$ with intersection number with some 2-sphere equal to $k \neq 0$, then $\left(q^{2 k}-1\right) K=0$ in $\mathcal{S}(M)$.

(ii) Let $K^{\prime}$ be a link in $M$ with intersection number with some torus equal to $k \neq 0$. Let $K$ be a link obtained from $K^{\prime}$ by adding to $K^{\prime}$ a non-contractible curve on the torus. Then $\left(q^{2 k}-1\right) K=0$ in $\mathcal{S}(M)$.

The results in (b) are proven in $(13,1.3)$ by explicit construction and (a) has been announced there. We will give a proof of Przytycki's theorem in section 5 . A similar and related skein module based on homology of framed links has been computed in [15]. This module has been described as the quantum deformation of the first homology group of $M$ in the same way as $\mathcal{S}(M)$ can be considered as the quantum deformation of the fundamental group of $M$. Note that the ring homomorphism $R \rightarrow 1$ mapping $q$ to 1 induces the $(R, \mathbb{Z})$-homomorphism

$$
\mathcal{S}(M) \rightarrow S \mathbb{Z} \hat{\pi}(M) .
$$

Here and in the following, we use the following notation. Let $\chi: \mathcal{R} \rightarrow \mathcal{R}^{\prime}$ be a fixed homomorphism of commutative rings with 1 . Then an $\left(\mathcal{R}, \mathcal{R}^{\prime}\right)$-homomorphism $\phi: \mathcal{M} \rightarrow \mathcal{M}^{\prime}$ from some $\mathcal{R}$-module into some $\mathcal{R}^{\prime}$-module is a homomorphism of abelian groups satisfying $\phi(\lambda m)=\chi(\lambda) \phi(m)$ for all $m \in \mathcal{M}$ and $\lambda \in \mathcal{R}$.

In this paper we will give a complete description of $\mathcal{S}(M)$, which is based on ideas from Vassiliev theory; see also [8, 5], [16. This settles Problem I. 92, Part I from Kirby's problem list [9]. We will reduce the understanding of relations in a natural presentation of $\mathcal{S}(M)$ to certain problems in the theory of singular 2-spheres and tori in $M$. The relation with Przytycki's result is based on D. Gabai's generalization of the sphere theorem relating singular with embedded surface theory [2].

For $r \geq 0$ let $\Lambda_{r}(M):=\operatorname{map}\left(\amalg_{r} S^{1}, M\right) / \Sigma_{r}$, where map denotes the set of smooth maps and the permutation group $\Sigma_{r}$ acts by permuting the circles in the domain. Note that $\Lambda_{1}(M)=: \mathbb{L}(M)$ is the space of smooth free loops in $M$ and $\Lambda_{0}(M)$ is a 1-point space by definition. Let $\Lambda(M):=\amalg_{r \geq 0} \Lambda_{r}(M)$ be the generalized free loop space of $M$. Let $I(M) \subset \Lambda(M)$ be the subspace of immersed maps.

We will prove the following two results:

Theorem 2. There exists a system of local coefficients $\mathfrak{Z}$ in $I(M)$, with $\mathfrak{Z}_{x} \cong R$ for $x \in I(M)$, such that

$$
H_{0}(I(M), \mathfrak{Z}) \cong \mathcal{S}(M) .
$$

The set $\pi_{0}(\mathbb{L}(M))$ is in 1-1 correspondence with $\hat{\pi}(M)$, and $\pi_{0}(\Lambda(M)) \cong \pi_{0}(I(M))$ is in 1-1 correspondence with the set $\mathfrak{b}(M)$ of unordered sequences in $\hat{\pi}(M)$. Also $\mathfrak{b}(M)$ is the natural basis of the module $S \mathcal{R} \hat{\pi}(M)$, which is isomorphic to the homology module $H_{0}(\Lambda(M), \mathcal{R})$ ( $\mathcal{R}$ any ring). In the following the elements of $\mathfrak{b}(M)$ will be identified with monomials in $\hat{\pi}(M)$.

Theorem 3. For each $\alpha \in \mathfrak{b}(M)$ there is a non-negative integer $\varepsilon(\alpha)$, which is determined by oriented intersection numbers in $M$, and an isomorphism of $R$-modules:

$$
\mathcal{S}(M) \cong \bigoplus_{\alpha \in \mathfrak{b}(M)} R /\left(q^{2 \varepsilon(\alpha)}-1\right) .
$$


The isomorphism of the theorem is determined by a choice of links with given free homotopy classes of components, which are called standard links. A precise description of the index $\varepsilon(\alpha)$ of $\alpha$ is given below.

Note that each 0-dimensional twisted homology module is a direct sum of cyclic modules with cyclic summands corresponding to path components [18. The theorems above provide a description of the relations in the skein module through monodromies of paths in $I(M)$. The skein module $\mathcal{S}(M)$ is free if and only if all monodromies are trivial, and $\mathcal{S}(M) \cong S R \hat{\pi}(M)$ in this case.

The image of a link $K$ in the skein module is determined by the free homotopy classes of its components (some element $\alpha \in \mathfrak{b}(M)$ determining a path component of $\Lambda(M)$ ) and some integer number $w(K)$, which is well-defined modulo $2 \varepsilon(\alpha)$. It follows from the skein relations that this number can be interpreted as the writhe (relative to a chosen standard link) of $K$. Thus, by computing the skein module, the indeterminancy of the universal writhe invariant is determined. But it should be noted that the writhe defined in this way does not satisfy the usual behaviour with respect to smoothing of crossings as known for $S^{3}$. This homological writhe of links is measured in the quantum deformation of the first homology group of $M$ [15].

Next we describe the index function $\varepsilon$. Throughout we will use the natural Hurewicz isomorphism from oriented singular bordism to oriented homology in dimensions 1 and 2 (see e.g. [7, 13.15). Let

$$
\lambda: H_{2}(M) \otimes H_{1}(M) \longrightarrow \mathbb{Z}
$$

be defined by the oriented intersection number of oriented (maybe singular) closed surfaces and loops in $M$. Let

$$
\mu: \mathfrak{b}(M) \rightarrow H_{1}(M)
$$

be defined by taking the sum of the homology classes resulting by application of the Hurewicz homomorphism to the free homotopy classes in $\alpha \in \mathfrak{b}(M)$. For $\beta \in \hat{\pi}(M)$ let $f_{\beta}: S^{1} \rightarrow M$ be a representing map. Let

$$
\widetilde{\mu}: \pi_{1}\left(\mathbb{L}(M), f_{\beta}\right) \rightarrow H_{2}(M)
$$

be defined by taking the homology class of the map $c^{\prime}: S^{1} \times S^{1} \rightarrow M$ adjoint to $c \in \pi_{1}\left(\mathbb{L}(M), f_{\beta}\right)$. Now for $\alpha=\alpha_{1} \cdot \ldots \cdot \alpha_{r} \in \mathfrak{b}(M)$ let $\Gamma(\alpha)$ be the subgroup of $\mathbb{Z}$ generated by all elements in

$$
\lambda\left(\widetilde{\mu}\left(\pi_{1}\left(\mathbb{L}(M), f_{\alpha_{i}}\right)\right), \mu(\alpha)\right)
$$

for all $1 \leq i \leq r$. If $\Gamma(\alpha)$ is nontrivial let $\varepsilon(\alpha)$ be a positive generator, otherwise let $\varepsilon(\alpha)$ be 0 . Note that $\varepsilon(\emptyset)=0$, where $\emptyset$ is the unique sequence in $\mathfrak{b}(M)$ of length 0 . Obviously $\varepsilon(\alpha)$ does not depend on the choice of maps $f_{\beta}$.

The following result can be considered as the singular version of Przytycki's theorem (a) (the proof also gives the idea for (b)):

Proposition 1. $\mathcal{S}(M)$ is isomorphic to $S R \hat{\pi}(M)$ (equivalently is free) if and only if each mapping from a torus to $M$ is homologous into $\partial M$.

Proof. If each mapping from a torus to $M$ is homologous into $\partial M$, then all oriented intersection numbers of closed curves and tori are trivial. Thus the index is trivial for each $\alpha \in \mathfrak{b}(M)$. Assume that there is a mapping from a torus, which is not homologous into $\partial M$. It follows by Poincare duality (see [6], Appendix A) that there exists an oriented loop, which has a non-trivial intersection number with the 
singular torus. The map from the torus is the adjoint map of a representative of some element in $\pi_{1}\left(\mathbb{L}(M), f_{\beta}\right)$ for some $\beta \in \hat{\pi}(M)$. Assume that the intersection number of the torus map and $f_{\beta}$ is non-trivial. Then each knot with homotopy class $\beta$ is torsion in $\mathcal{S}(M)$ by Theorem 2 . Otherwise we can find $\beta^{\prime} \in \hat{\pi}(M)$ such that $\beta^{\prime}$ has a non-trivial intersection number with the torus map. Then for $\alpha:=\beta \cdot \beta^{\prime}$ we have $\varepsilon(\alpha) \neq 0$. Thus $\mathcal{S}(M)$ has torsion.

\section{Statement of the General Results}

The quantum deformation of the fundamental group determines the writhe invariants of links in oriented 3 -manifolds in a universal way. The writhe of a link in $S^{3}$ naturally is the sum of the self-writhe and the total linking number (sum of pairwise linking numbers). Accordingly one can define a skein module of oriented 3 -manifolds, which contains the self-writhe and total linking number invariants. It turns out that the computation of this module does not require ideas beyond those used for the computation of the module $\mathcal{S}(M)$.

Let $R^{\prime}:=\mathbb{Z}\left[q_{1}^{ \pm 1}, q_{2}^{ \pm 1}\right]$. Let $\mathcal{S}^{\prime}(M)$ be the quotient of $R^{\prime} \mathfrak{L}(M)$ by the submodule generated by all elements of the form $K_{+}=q_{1}^{2} K_{-}$, respectively $K_{+}=q_{2}^{2} K_{-}$, for crossings of the same, respectively distinct, components, and $K^{(1)}=q_{1} K$. Using the coefficient homomorphism $R^{\prime} \rightarrow R$, which maps $q_{1}$ and $q_{2}$ to $q$, Przyztycki's universal coefficient theorem [14] induces the natural isomorphism

$$
\mathcal{S}(M) \cong \mathcal{S}^{\prime}(M) \otimes_{R^{\prime}} R \text {. }
$$

Similarly, the coefficient homomorphism $R^{\prime} \rightarrow R$, which maps $q_{1}$ to 1 and $q_{2}$ to $q$, induces the homomorphism onto the linking number skein module $\mathcal{L}(M)$ considered in [5]. Also there is the natural homomorphism of skein modules defined from the ring homomorphism $R^{\prime} \rightarrow R$, which maps $q_{2}$ to 1 and $q_{1}$ to $q$. Let $\mathcal{W}(M)$ denote the resulting skein module. In this skein module the relative self-writhe of links is measured. The above modules can be summarized in the following diagram of natural $\left(R^{\prime}, R\right)$-epimorphisms:

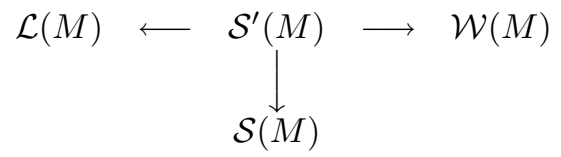

The modules $\mathcal{L}(M)$, respectively $\mathcal{W}(M)$, contain the link homotopy, respectively anti-link homotopy, part of $\mathcal{S}^{\prime}(M)$ in a natural way.

The following two results are immediate generalizations of Theorems 2 and 3 . Let $\mathbb{N}$ denote the set of non-negative integers.

Theorem 4. There exists a system of local coefficients $\mathfrak{Z}^{\prime}$ in $I(M)$ with $\mathfrak{Z}_{x}^{\prime} \cong R^{\prime}$ such that

$$
H_{0}\left(I(M), \mathfrak{Z}^{\prime}\right) \cong \mathcal{S}^{\prime}(M) .
$$

Theorem 5. For each $\alpha \in \mathfrak{b}(M)$ there exists an index

$$
\varepsilon^{\prime}(\alpha)=\left(\varepsilon_{1}(\alpha), \varepsilon_{2}(\alpha), \varepsilon_{3}(\alpha) \in \mathbb{N} \times \mathbb{Z} \times \mathbb{N},\right.
$$

determined by oriented intersection numbers as described below, such that

$$
\mathcal{S}^{\prime}(M) \cong \bigoplus_{\alpha \in \mathfrak{b}(M)} R^{\prime} /\left(q_{1}^{2 \varepsilon_{1}(\alpha)} q_{2}^{2 \varepsilon_{2}(\alpha)}-1, q_{1}^{2 \varepsilon_{3}(\alpha)}-1\right) .
$$


The numbers $\varepsilon^{\prime}(\alpha)$ are defined as follows. For $\alpha=\alpha_{1} \ldots \alpha_{r}$ and $f_{\alpha_{i}}$ representing $\alpha_{i}$, let $\Gamma^{\prime}(\alpha)$ be the subgroup of $\mathbb{Z} \times \mathbb{Z}$ generated by all elements (omitting the Hurewicz homomorphisms from the notation)

$$
\left(\lambda\left(c, \alpha_{i}\right), \lambda\left(c, \alpha \backslash \alpha_{i}\right)\right),
$$

where $c$ runs through $\pi_{1}\left(\mathbb{L}(M), f_{\alpha_{i}}\right)$ and $1 \leq i \leq r$. Then $\Gamma^{\prime}(\alpha)$ is generated by two elements, which we can write $\left(\varepsilon_{1}(\alpha), \varepsilon_{2}(\alpha)\right)$ and $\left(\varepsilon_{3}(\alpha), 0\right)$ with $\varepsilon_{1}(\alpha), \varepsilon_{3}(\alpha) \in \mathbb{N}$. $\left(\Gamma^{\prime}(\alpha)\right.$ is cyclic if and only if $\varepsilon_{3}(\alpha)=0$.)

It follows from the skein relations that the image of a given link $K$ in $\mathcal{S}^{\prime}(M)$ can be written as $q_{1}^{w_{1}(K)} q_{2}^{w_{2}(K)}$ multiplied by a standard link with homotopy classes determined by $\alpha \in \mathfrak{b}(M)$. Here $w_{1}(K)$ and $w_{2}(K)$ are the relative self-writhe and relative total linking number of $K$. The pair $\left(w_{1}(K), w_{2}(K)\right) \in \mathbb{Z}^{2}$ is welldefined modulo the subgroup $\Gamma^{\prime}(\alpha)$. Obviously the sum of the total linking number and the self-writhe is the writhe number in Theorem 3. The number $\varepsilon_{2}(\alpha)$ is the linking number index as defined in [5]. The $g c d$ of $\left(\varepsilon_{1}(\alpha)+\varepsilon_{2}(\alpha)\right)$ and $\varepsilon_{3}(\alpha)$ is the indeterminancy $\varepsilon(\alpha)$ as defined in section 1 .

Proposition 2. $\mathcal{S}^{\prime}(M)$ is isomorphic to $S R^{\prime} \hat{\pi}(M)$ if and only if each mapping from a torus to $M$ is homologous into $\partial M$.

Proof. The "only if" part follows from Przytycki's universal coefficient theorem and Proposition 1. If every singular torus is homologous into $\partial M$, then all intersection numbers of singular tori and loops are zero. So the subgroups $\Gamma^{\prime}(\alpha) \subset \mathbb{Z} \times \mathbb{Z}$ are trivial for all $\alpha$. It follows that $\mathcal{S}^{\prime}(M)$ is free.

Remark 1. In [1], using the language of Vassiliev invariants, the module $\mathcal{W}(M)$ has been discussed. It follows from our results that this module is free if and only if the following condition holds: For each singular torus, all intersection numbers with loops defined by restriction of the torus map to essential closed curves on the torus are trivial. In this case the relative framing number of a framed knot in $M$ is a well-defined integer (since the linking number of parallel curves is well-defined). In particular the framing of a knot cannot be changed by isotopy. In fact, it is known that the framing of a knot can be changed by isotopy if and only if $M$ contains a non-separating 2-sphere. This follows immediately from work of D. McCullough as pointed out in [4]; see also [1].

\section{Skein Relations ARE MONODROMies}

In this section we prove Theorem 4. The arguments used here are similar to those in [5].

For details concerning bundles of groups and homology with twisted coefficients we refer to [18], pp. 257-290. Recall that the fundamental groupoid of a space $B$ is a category $\Pi_{1}(B)$ whose objects are points of $B$ and whose morphisms $b_{1} \rightarrow b_{2}$ are defined by the set of homotopy classes of paths in $B$ from $b_{1}$ to $b_{2}$. Then a bundle of abelian groups (or abelian local coefficient system) is a functor $\mathfrak{G}$ from $\Pi_{1}(B)$ into the category of abelian groups. Thus for each $b \in B$ there is defined the abelian group $\mathfrak{G}_{b}$. In [18], pp. 265-266, the chain groups, boundary operators and twisted homology groups $H_{*}(B ; \mathfrak{G})$ are defined. It is proved in [18], Theorem 1.12 that for $B$ a connected space and a given operation of $\pi_{1}\left(B, b_{0}\right)$ on some abelian group $\mathfrak{G}_{0}$, there is defined a bundle of groups in $B$ inducing the given action of the fundamental group. Also in [18], Theorem 3.2, the 0-dimensional twisted homology 
with coefficients in $\mathfrak{G}$ is described explicitly. In fact, $H_{0}(B ; \mathfrak{G}) \cong \mathfrak{G}_{0} / \mathfrak{H}_{0}$, where $\mathfrak{H}_{0}$ is the subgroup of $\mathfrak{G}_{0}=\mathfrak{G}_{b_{0}}$ generated by all monodromy elements of the from $x-\xi x$ with $x \in \mathfrak{G}_{0}$ and $\xi \in \pi_{1}\left(B, b_{0}\right)$.

We will apply the definitions and results from above to the space $B=I(M)$ of immersions in $M$. The local coefficient system will be constructed from the skein relation.

Recall that elements of $I(M)$ are unordered oriented immersions of circles in $M$. For a given element $x \in I(M)$ consider all unordered framed immersions with the same underlying oriented immersion $x$. We will consider two such immersions equivalent if either

(i) their framings are homotopic, or

(ii) if they differ by twists in the framings of the components, such that the total signed number of these twists is zero.

A resulting equivalence of framed immersions is called a total framing of $x$. We define $\mathfrak{Z}_{x}^{\prime}$, respectively $\mathfrak{Z}_{x}$, by the free $\mathbb{Z}\left[q_{2}^{ \pm}\right]$-module, respectively by the free abelian group, generated by all total framings of $x$. Note that there is a transitive action of $\mathbb{Z}$ on the set of total framings of $x$ for each $x \in I(M)$, and $\mathfrak{Z}_{x}^{\prime} \cong R^{\prime}$, respectively $\mathfrak{Z}_{x} \cong R$, for each $x \in I(M)$.

There is an obvious notion of isotopy of totally framed links in $M$. Let $\mathfrak{T}(M)$ denote the corresponding set of isotopy classes. It is immediate from the definitions that the skein module $\mathcal{S}^{\prime}(M)$ can be defined from the set $\mathfrak{T}(M)$ using the same relations as in the original definition of $\mathcal{S}^{\prime}(M)$. Note that the total framing of the link $K^{(1)}$ is given by the action of +1 on the total framing of $K$.

The collection of modules $\mathfrak{Z}_{x}^{\prime}$ forms a bundle of modules in $I(M)$. The corresponding local system of coefficients in $I(M)$ is defined by describing, for each $\alpha \in \mathfrak{b}(M)$, the action of $\pi_{1}(I(M), x)$ on $\mathfrak{Z}_{x}^{\prime}$. Here $x=x_{\alpha}$ is a link with free homotopy classes of components given by $\alpha \in \mathfrak{b}(M)$.

Let $\overline{I(M)} \rightarrow I(M)$ be the space of ordered immersions of circles in $M$. Then the projections $\widetilde{I(M)} \rightarrow I(M)$ (and similarly $\widetilde{\Lambda(M)} \rightarrow \Lambda(M)$ ) are covering maps away from the fixed point sets of the permutation actions. We will have to choose the base points $x=x_{\alpha}$ in a specific way. If $\alpha$ repeatedly contains a conjugacy class, then we can choose $x_{\alpha}$ to be symmetric in the following way: All components with the same free homotopy class will be parallel to each other with respect to some framing. It follows that there are isotopies (supported in a neighboorhood of the link) which change the order of those components. The loops in $I(M)$ defined in this way are called small. Note that by composition with loops defined by these isotopies each loop in $I(M)$ can be assumed to lift to a loop in $\widehat{I(M)}$. Note that the bundle of modules pulls back to the covering space in the obvious way.

For $\gamma \in \pi_{1}(I(M), x)$ let $\tilde{\gamma}$ be the loop, which results, possibly after composition with a small loop, by lifting to $\widetilde{I(M)}$. We choose a total framing on $x$ and lift this total framing to the corresponding basepoint on $\tilde{\gamma}$. It follows from Hirsch theory 3] that this total framing canonically transports along $\tilde{\gamma}$ and defines a new total framing on $x$. It is easily seen to be well-defined and independent of all choices made. The difference to the original framing is determined by some element $k_{1}(\gamma) \in \mathbb{Z}$.

Next perturb the loop $\tilde{\gamma}$ to be transverse so that all singular maps along the path are immersions with a single double point. Let $k_{2}(\gamma)$, respectively $k_{3}(\gamma)$, be the signed sum of the singular points along $\gamma$ (or $\tilde{\gamma}$ ) (i.e. positive, respectively 
negative, crossing changes), where the double point of the immersion is a selfcrossing, respectively a crossing of distinct components.

Theorem 6. The collection of homomorphisms (of groups)

$$
k=k_{\alpha}: \pi_{1}\left(I(M), x_{\alpha}\right) \rightarrow \operatorname{Aut}\left(R^{\prime}\right),
$$

defined by: $k(\gamma)$ is multiplication by

$$
q_{1}^{k_{1}(\gamma)+2 k_{2}(\gamma)} q_{2}^{2 k_{3}(\gamma)},
$$

is well-defined, and thus defines a system of local coefficients $\mathfrak{Z}^{\prime}$ in $I(M)$.

Proof. We have to show that the maps $k$ are constant under homotopies of loops in $I(M)$. The homomorphism property follows easily from the definitions. We will show more generally that $k$ is well-defined on $\pi_{1}(\Lambda(M), x)$. (This will be important later on.) We can assume that $\gamma$ lifts to a loop in $\widetilde{I(M)}$ and consider a loop $\gamma^{\prime}$ homotopic to $\gamma$. The homotopy lifts to a homotopy in $\widetilde{I(M)}$, and we can apply Lin's transversality results [12] and change the homotopy to a transversal position with respect to the complex of singular links in $M$. (It is important that this approximation be done relative to the boundary. This can easily be seen by following the arguments of Lin's proof.) Then the preimage of the set of singular links is a 1-dimensional complex in the domain of the homotopy (annulus), with vertices in the boundary and interior vertices of valence 4 or 1 . Those of valence 4 describe immersions with two double points, those of valence 1 describe kink resolutions (for further details see [ $[8]$ or [5]). In order to show that the contributions from the two loops are equal, deform one into the other across the annulus in a finite number of steps, crossing only over a single interior vertex at a time. At maxima or minima the contributions cancel obviously. It is easy to see that $k$ does not change when crossing over a vertex of valence 4 (the corresponding contribution around each vertex is zero). If we cross over a vertex of valence 1 we encounter a crossing change, which does not change the oriented isotopy type but does change the total framing. The framing change cancels the singularity contribution. Thus crossing over any vertex does not change the value of $k$.

By Theorem 6 the homology module $H_{0}\left(I(M), \mathfrak{Z}^{\prime}\right)$ is well defined. In Theorem 7 we will define the isomorphism with $\mathcal{S}^{\prime}(M)$. Recall that, as a twisted 0-dimensional homology module, $\mathcal{S}^{\prime}(M)$ is a direct sum of cyclic modules $R^{\prime} / \mathfrak{I}(\alpha)$, where $\alpha$ runs through the set of path components of $I(M)$ (which is in 1-to-1 correspondence with $\mathfrak{b}(M))$. The ideals $\mathfrak{I}(\alpha)$ are generated by all elements of the form $k(\gamma)-1$, where $\gamma$ runs through all elements of $\pi_{1}\left(\Lambda(M), f_{\alpha}\right)$ (compare e.g. [18, Theorem 3.2). Thus the proofs of both Theorems 5 and 3 reduce to the problem of relating the monodromies to intersection numbers as explained in sections 1 and 2 . This will be done in section 4 .

Theorem 7. There is a natural isomorphism

$$
\mathcal{S}^{\prime}(M) \longrightarrow H_{0}\left(I(M), \mathfrak{Z}^{\prime}\right) \text {. }
$$

Proof. There is the obvious map $\mathfrak{T}(M) \rightarrow H_{0}\left(I(M), \mathfrak{Z}^{\prime}\right)$, defined by mapping a link to the 0 -chain represented by a representative link $x$. Note that the framing determines an element in the fibre over $x$. So we have defined a 0 -cycle for homology with twisted coefficients; see [18], p. 266. Recall that $H_{0}\left(I(M), \mathfrak{Z}^{\prime}\right)$ is an $R^{\prime}$-module 
(this can be seen e.g. from the definition as a homology group of the chain complex $C_{*}(\overline{I(M)}) \otimes R^{\prime}$, where $\overline{I(M)}$ is the universal covering space of $I(M)$, and the tensor product is over the action of the fundamental group defining the system of local coefficients). The map above extends uniquely to an $R^{\prime}$-homomorphism $R^{\prime} \mathfrak{T}(M) \rightarrow H_{0}\left(I(M), \mathfrak{Z}^{\prime}\right)$. This homomorphism is onto because every 0 -cycle can be represented, up to homology, by a link in $M$. It follows from the very definitions that skein equivalent links are homologous in the twisted homology module. Thus there is an induced epimorphism from the skein module into the twisted homology module. This epimorphism is injective by the definition of the boundary operator for the chain groups defined by the system of local coefficients (see [18, p. 274).

\section{Computation of the monodromies}

Recall from the proof of Theorem 6 that $k(\gamma)$ only depends on the homotopy class of $\gamma$ in $\Lambda(M)$. Moreover, by using the small loops of embeddings, which change the order of components (described before Theorem 6), we can assume that $\gamma$ actually lifts to a loop in $\widetilde{\Lambda(M)}$. But $\pi_{1}\left(\widetilde{\Lambda(M)},\left(f_{1}, \ldots, f_{r}\right)\right)$ is isomorphic to the direct product of groups $\pi_{1}\left(\mathbb{L}(M), f_{i}\right)$. Because of the homomorphism property of $k$ (see Theorem 6) it suffices to compute $k(\gamma)$ on those loops in $I(M)$, which fix all but one component. For such a special loop in $I(M)$ let $\alpha_{i}$ be the free homotopy class of that component, which actually is moved in $M$ along $\gamma$. As before let $\alpha_{i}$ be represented by $f_{\alpha_{i}}: S^{1} \rightarrow M$. We can assume that $f_{\alpha_{i}}$ is an embedding. Let $c \in \pi_{1}\left(\mathbb{L}(M), f_{\alpha_{i}}\right)$ be a loop in the free loop space based in $f_{\alpha}$. Obviously the loop $\gamma$ in $I(M)$ is completely determined by $c$ and the components $f_{j}, j \neq i$, which are fixed during the deformation. Note that the indices, which have to be computed, only depend on the homology classes of the maps $f_{i}$.

Next consider the trace of the moving component of such a loop. If we assume that the underlying loop in the mapping space is transverse as described in section 3 , then the resulting immersion from $S^{1} \times I$ to $M \times I$ is self-transverse; compare [1]. Moreover by a reparametrization we can assume that the loop is constant near $S^{1} \times\{0,1\}$. Thus the immersions over the two boundary components can be glued together to yield a self-transverse immersion

$$
f: S^{1} \times S^{1} \rightarrow M \times S^{1} .
$$

(Here we use that $f_{\alpha_{i}}$ is an embedding.) A framing of the embedding $f_{\alpha_{i}}: S^{1} \rightarrow M$ defines a framing of the normal bundle of the immersion $S^{1} \times I \rightarrow M \times I$ over $S^{1} \times\{0\}$. This framing extends to a framing of the immersion $S^{1} \times I \rightarrow M \times I$. But the resulting framing of the restriction to $S^{1} \times\{1\}$ can differ from the one given over $S^{1} \times\{0\}$ (while the immersions on the two ends of the cylinder agree). Thus we have defined a normal framing of the self-transverse immersion $f$ except along the gluing circle. The difference between the framings on the top and bottom is precisely given by $k_{1}(\gamma)$ and is the normal Euler number $\chi(f)$ of the immersion. This is well known from the singularity interpretation of the normal Euler number by using a section of the 2-dimensional normal bundle, which is generic with respect to the zero-section (compare e.g. [10], pp. 33-34).

Then $k(\gamma)$ is obviously computed from the self-intersections of this immersion and from the intersection numbers of the projection to $M$ with the constant components. The oriented intersection numbers of the torus in $M$ with the other components determine $k_{3}(\gamma)$. So we only have to consider the immersed torus. 
It is a classical result, in its original version due to Whitney 19] (discussed e.g. in the work of Lashof and Smale [11]), that the homological intersection number of $[f] \in H_{2}\left(M \times S^{1}\right)$ satisfies the following relation:

$$
[f] \cdot[f]=2 D(f)+\chi(f)
$$

Here $D(f)$ is the oriented self-intersection number of the self-transverse immersion $f$, which is equal to $k_{2}(\gamma)$ by definition.

Thus the proof of Theorem 5 is complete by proving

Lemma 1. The homological intersection number $[f] \cdot[f]$ is equal to $2 \lambda\left(c, \alpha_{i}\right)$.

It follows that

$$
2 \lambda\left(c, \alpha_{i}\right)=[f] \cdot[f]=k_{1}(\gamma)+2 k_{2}(\gamma) .
$$

By considering corresponding powers of $q_{1}$ (respectively $q_{2}$ ) we see that $\varepsilon^{\prime}$ can be computed as described in Theorem 5.

Proof of Lemma 1. Using the Künneth theorem

$$
H_{2}\left(M \times S^{1}\right) \cong H_{2}(M) \oplus\left(H_{1}(M) \otimes H_{1}\left(S^{1}\right)\right)
$$

we will identify $H_{2}(M)$ and $H_{1}(M) \otimes H_{1}\left(S^{1}\right)$ with subgroups of $H_{2}\left(M \times S^{1}\right)$. Next define $g: S^{1} \times S^{1} \rightarrow M \times S^{1}$ by $g(x, t)=(f(x, 1), t)$ and let $a:=[g] \in$ $H_{1}(M) \otimes H_{1}\left(S^{1}\right)$. Then an easy bordism argument shows that $[f]-a=: b \in H_{2}(M)$ is equal to $\widetilde{\mu}(c)$, and $[f]=a+b$ is the decomposition of $[f]$ according to the Künneth

theorem. Note that $a \cdot a=b \cdot b=0$ (intersection numbers in $M \times S^{1}$ ). Thus $[f] \cdot[f]=2 a \cdot b$. But $a \cdot b$ is also the oriented intersection number of $\widetilde{\mu}(c) \in H_{2}(M)$ with the homology class represented by $f \mid\left(S^{1} \times\{1\}\right)$.

\section{Computational tools and proof of Przytycki's theorem}

Let $\Omega M$ be the based loop space of $M$. Consider the fibration

$$
\Omega M \stackrel{i}{\rightarrow} \mathbb{L} M \stackrel{p}{\rightarrow} M
$$

where $i$ is the inclusion and $p$ is the evaluation at the basepoint (see [17] and [5]). For given $b \in \pi_{1}(M)$ let $f_{b}$ be a basepoint in $\Omega(M)$ corresponding to $b$ and let $\beta \in \hat{\pi}(M)$ be the corresponding free homotopy class.

There is the exact sequence of homotopy groups:

$$
\pi_{1}\left(\Omega M, f_{b}\right) \stackrel{i_{*}}{\longrightarrow} \pi_{1}\left(\mathbb{L} M, f_{b}\right) \stackrel{p_{*}}{\longrightarrow} \pi_{1}(M) \stackrel{[, b]}{\longrightarrow} \pi_{1}(M) .
$$

Note that there are isomorphisms $\pi_{1}\left(\mathbb{L}(M), f_{b}\right) \cong \pi_{1}\left(\mathbb{L} M, f_{\beta}\right)$, where the map $f_{\beta}: S^{1} \rightarrow M$ is the map chosen in section 1 , and $[, b]$ denotes the commutator with $b$.

There is an obvious isomorphism $\pi_{2}(M) \cong \pi_{1}\left(\Omega M, f_{b}\right)$. (Deform a loop in $f_{b}$ in $\Omega(M)$ such that all the mappings $S^{1} \rightarrow M$ along the loop are constant on a chosen neighbourhood of the basepoint. We can assume that $f_{b}$ has this property. Then the obstruction to the constant loop in $f_{b}$ is uniquely determined by some element in $\pi_{2}(M)$.)

Now the image $i_{*}(c)$ for $c \in \pi_{2}(M)$ can be described as follows. Represent $c$ by a mapping of a 2-sphere $g$, which is transverse to $f_{b}$. Now deform the restriction of $f_{b}$ on a neighbourhood of the basepoint in $* \in S^{1}$ as follows: Choose an arc joining $f_{b}(*)$ with $g(*)$. Then deform $f_{b}$ along this arc until a small neighbourhood of $*$ is mapped to $g\left(S^{2}\right)$. Now deform across the image of the 2 -sphere in the obvious way. 
This is a description of $i_{*}(c) \in \pi_{1}\left(\mathbb{L} M, f_{b}\right)$. Note that if the intersection number of $f_{b}$ and $g$ is equal to $k \in \mathbb{Z}$, then

$$
\lambda\left(\widetilde{\mu}\left(i_{*}(c)\right), \mu\left(f_{b}\right)\right)=k .
$$

Note that this intersection number is trivial for all 2-sphere mappings, which are homotopic to $\partial M$.

Example 1. Let $M=S^{2} \times S^{1}$. Then $\hat{\pi}(M)=H_{1}(M) \cong \mathbb{Z}$ and $\mathfrak{b}(M)$ can be identified with the set of unordered sequences of integer numbers. Note that $p_{*}$ is onto and $\pi_{1}\left(\mathbb{L} M, f_{b}\right)$ is the union of sets $p_{*}^{-1}\left(b^{\prime}\right)$ with a transitive action of $\pi_{2}(M)$ on each of these sets.

Since $\pi_{1}(M)$ is cyclic, a mapping from a torus (representing some element $x \in$ $\left.\pi_{1}\left(\mathbb{L} M, f_{b}\right)\right)$ can be homotoped to a map $f$ so that the restriction to the 1-skeleton $S^{1} \vee S^{1}$ maps into the core $* \times S^{1}$. Now this restriction can be extended to a map of a torus into $* \times S^{1}$. Thus the collection of intersection numbers $\lambda(\widetilde{\mu}(x), \mu(\alpha))$, where $x$ runs through all of $p^{-1}\left(b^{\prime}\right)$, does not depend on $b^{\prime}$. Here $\alpha \in \mathfrak{b}(M)$ is any sequence of free homotopy classes. So we can restrict the computation to $b^{\prime}=0$ and compute all intersection numbers from the singular tori resulting from elements in the image of $i_{*}$.

Note that $\pi_{2}(M)$ is generated by multiples of the standard sphere $S^{2} \times *$. Moreover, a loop in $M$ has homology class $k \in \mathbb{Z}$ if and only if the loop intersects $S^{2} \times *$ with intersection number $k$. It follows that for $\alpha \in \mathfrak{b}(M)$

$$
\varepsilon(\alpha)=\varepsilon\left(\alpha_{1} \cdot \ldots \cdot \alpha_{r}\right)=\sum_{i=1}^{r} \alpha_{i} .
$$

The subgroup $\Gamma(\alpha)$ of $\mathbb{Z} \times \mathbb{Z}$ is generated by the elements $\left(\alpha_{i}, \sum_{j \neq i} \alpha_{j}\right)$. So if we consider $\alpha=1 \cdot 1 \cdot \ldots \cdot 1 \in \mathfrak{b}_{r}(M)$, we have the contributions $(1, r-1)$ for all $i$. Then $\varepsilon^{\prime}(\alpha)=(1, r-1,0)$ and the corresponding cyclic summand of the skein module is $R^{\prime} /\left(q_{1}^{2} q_{2}^{2(r-1)}-1\right)$. For $\alpha=1 \cdot 2$ the resulting subgroup of $\mathbb{Z} \times \mathbb{Z}$ is generated by $(1,2)$ and $(2,1)$. The summand of the skein module corresponding to this $\alpha$ is given by $R^{\prime} /\left(q_{1}^{4} q_{2}^{2}-1, q_{1}^{6}-1\right)$. Even though the computation is easy for a given $\alpha$, a general answer might involve some interesting combinatorics.

Finally we give the proof of Theorem 1 from Theorem 2 .

First we show (b). Note that $\lambda\left(\widetilde{\mu}\left(i_{*}(c)\right), \mu(K)\right)=k$ for $K$ a knot in $M$, which intersects the 2-sphere (with homotopy class $c \in \pi_{2}(M)$ ) with intersection number $k \in \mathbb{Z}$. This shows (i). To prove (ii) we define the element $c \in \pi_{1}\left(\mathbb{L} M, f_{\beta}\right)$, where $f_{\beta}: S^{1} \rightarrow M$ is a representative of the given non-contractible curve on the torus with free homotopy class $\beta \in \hat{\pi}(M)$, by a deformation of the non-contractible curve across the torus and back into itself. Then let $\alpha=\beta \cdot \gamma$, where $\gamma \in \hat{\pi}(M)$ is the free homotopy class of $K^{\prime}$. It follows that $\lambda(\widetilde{\mu}(c), \mu(\alpha))=k$, because pushing the non-contractible curve away from the embedded torus shows that the intersection number of $\gamma$ with $c$ is zero. This proves (ii).

In order to prove part (a) of Przytycki's result we have to show that $\varepsilon(\alpha)=0$ if there are no non-separating embedded 2-spheres or tori in $M$. Conversely we claim that $\varepsilon(\alpha) \neq 0$ implies the existence of a non-separating embedded 2-sphere or torus. Now it is immediate from the definitions that there exists $\alpha \in \mathfrak{b}(M)$ satisfying $\varepsilon(\alpha) \neq 0$ if and only if there are a singular torus and an oriented loop in $M$, which have a non-trivial intersection number. Note that each singular 2sphere, by composition with the canonical projection $S^{1} \times S^{1} \rightarrow S^{2}$, induces a 
singular torus, preserving intersection numbers with a loop. Obviously (see e.g. [6], Appendix A) each oriented embedded surface with a non-trivial intersection number with an oriented loop is non-separating.

Thus Przytycki's theorem follows easily from the following consequence of D. Gabai's fundamental result.

Theorem 8. Suppose that $M$ is a compact oriented 3-manifold and let $\gamma$ be an oriented loop in $M$. If there is a singular torus in $M$ with non-trivial intersection number with $\gamma$, then there also exists an embedded 2-sphere or torus with non-trivial intersection number with $\gamma$.

Proof. We apply Corollary 6.18 from $\left[2\right.$ to the homology class $z \in H_{2}(M)$ determined by the singular torus. It follows that the singular Thurston norm of $z$ is zero, so by Gabai's result also the embedded Thurston norm of $z$ is zero. It follows that $z$ can be represented by a disjoint union $T$ of embedded 2 -spheres and tori. This representative of $z$ has the same intersection number with $\gamma$ as the original singular torus. Obviously there is at least one component of $T$ which has non-trivial intersection number with $\gamma$.

\section{ACKNOWLEDGEMENTS}

It is a pleasure to thank J. Przytycki for bringing the problem discussed in this paper to my attention, and for many stimulating discussions and generous hospitality. Also I would like to thank Vladimir Chernov for helpful comments, and for pointing out his related work [1].

\section{REFERENCES}

[1] V. Chernov, Framed knots in 3-manifolds preprint math.GT/0105139, http://xxx.lanl.gov, 2001.

[2] D. Gabai, Foliations and the topology of 3-manifolds, J. Differential Geometry 18, 1983, 445-503. MR 86a:57009

[3] M. W. Hirsch, Immersions of manifolds Transactions of the AMS 93, 1959, 242-276. MR 22:9980

[4] J. Hoste, J. Przytycki, Homotopy skein modules of orientable 3-manifolds, Math. Proc. Camb. Phil. Soc. (1990), 108, 475-488. MR 91g:57006

[5] U. Kaiser, Presentations of q-homotopy skein modules of oriented 3-manifolds, Journal of Knot Theory and its Ramifications, no. 3, 2001, 461-491. MR 2002g:57011

[6] U. Kaiser, Link theory in manifolds, Lecture Notes in Mathematics 1669, Springer Verlag 1997. MR 98j:57010

[7] L. Kauffman, On Knots, Annals of Mathematical Studies 115, Princeton University Press 1987. MR 89c:57005

[8] E. Kalfagianni, X. S. Lin, The HOMFLY polynomial for links in rational homology spheres, Topology 38, no. 1, 1999, 95-115. MR 99h:57011

[9] R. Kirby. Problems in low dimensional topology, in 1993 Georgia International Topology Conference Proceedings, Part II, ed. by W. Kazez, AMS/IP Studies in Advanced Mathematics 1997.

[10] U. Koschorke, Vector Fields and Other Vector Bundle Morphisms - A Singularity Approach, Lecture Notes in Mathematics 847, 1981. MR 82i:57026

[11] R. K. Lashof, S. Smale, Self-intersections of immersed manifolds, Journal of Mathematics and Mechanics, Vol. 8, No. 1, 1959, 143-157. MR 21:332

[12] X. S. Lin, Finite type link invariants of 3-manifolds, Topology 33, no. 1, 1994, 45-71. MR 94m:57020

[13] J. Przytycki, Algebraic topology based on knots: an introduction, in Proceedings of Knots 96, edited by Shin'ichi Suzuki, 1997, 279-297. MR 99k:57030 
[14] J. Przytycki, Skein modules of 3-manifolds, Bull. Ac. Pol. Math. 39(1-2), 1991, 91-100. MR 94g:57011

[15] J. Przytycki, A q-analogue of the first homology group of a 3-manifold, Contemporary Mathematics Volume 214, 1998, 135-143. MR 98m:57011

[16] V. Vassiliev, On invariants and homology of spaces of knots in arbitrary manifolds, American Math. Soc. Translations (2), Vol. 185, 1998. MR 2000j:57030

[17] V. Vassiliev, Complements of discriminants of smooth maps: topology and applications, Transl. Math. Monographs, vol. 98, AMS, Providence, RI 1994. MR 94i:57020

[18] G. W. Whitehead, Elements of Homotopy Theory, Graduate Texts in Mathematics Vol. 61, Springer Verlag 1978. MR 80b:55001

[19] H. Whitney, The self-intersections of a smooth n-manifold in 2n-space, Ann. Math. (1944), 220-240. MR 5:273g

Department of Mathematics, Boise State University, 1910 University Drive, Boise, IDAHO 83725-1555

E-mail address: kaiser@math.boisestate.edu 